\title{
Effect of Grade and Surface Type on Peak Tibial Acceleration in Trained Distance Runners
}

\author{
Nathan Waite, ${ }^{1}$ John Goetschius, ${ }^{2}$ and Jakob D. Lauver ${ }^{3}$ \\ ${ }^{1}$ Adrian College; ${ }^{2}$ James Madison University; ${ }^{3}$ Coastal Carolina University
}

\begin{abstract}
Runners experience repeated impact forces during training, and the culmination of these forces can contribute to overuse injuries. The purpose of this study was to compare peak vertical tibial acceleration (TA) in trained distance runners on 3 surface types (grass, asphalt, and concrete) and 3 grades (incline, decline, and level). During visit 1, subjects completed a 1-mile time trial to determine their pace for all running trials: $80 \%(5 \%)$ of the average time trial velocity. During visit 2, subjects were outfitted with a skin-mounted accelerometer and performed 18 separate running trials during which peak TA was assessed during the stance phase. Each subject ran 2 trials for each condition with 2 minutes of rest between trials. Peak TA was different between decline (8.04 [0.12] g) and incline running $(7.31[0.35] \mathrm{g} ; P=.020)$. On the level grade, peak TA was greater during grass $(8.22[1.22] \mathrm{g})$ compared with concrete $(7.47$ [1.65] g; $P=.017)$. On the incline grade, grass $(7.68[1.44] \mathrm{g})$ resulted in higher peak TA than asphalt $(6.99[1.69] \mathrm{g} ; P=.030)$. These results suggest that under certain grade conditions grass may result in higher TA compared with either concrete or asphalt.
\end{abstract}

Keywords: skin-mounted accelerometers, impact acceleration, grass, concrete, asphalt

It is estimated that between $27 \%$ and $70 \%$ of all runners sustain a lower-extremity overuse injury during any 1-year training period, ${ }^{1-3}$ with fatigue fractures of the tibia occurring commonly in runners. ${ }^{4}$ One factor that contributes to overuse injuries is the repeated impact forces experienced with each foot strike, which can be amplified during times of increased training, possibly further contributing to the development of overuse injuries. ${ }^{5}$ It has been estimated that runners training a modest 20 miles a week for a year will collide with the ground more than 1.3 million times a year. ${ }^{6}$

Impact forces result from the collision of the foot with the ground during which the velocity of the foot decelerates to 0 , resulting in the generation of ground reaction forces (GRFs). ${ }^{7}$ In an effort to reduce the loads experienced by the joints as a result of these GRFs, the impact forces are attenuated by passive and active mechanisms ${ }^{4,8}$; however, additional factors, such as running velocity, surface type, and footwear, can affect these GRFs. ${ }^{4}$ In addition to the direct assessment of these GRFs, a moderate correlation between vertical impact forces and tibial acceleration (TA) recorded from skin-mounted accelerometers have been reported. ${ }^{9}$ These accelerometers can be used as a proxy for impact forces via Newton's second law and allow for assessments in real-world settings outside of the laboratory. The peak TA at foot contact can range from 2.98 to $10.5 \mathrm{~g}$ during various running velocities, ${ }^{10,11}$ and the likelihood of the history of a tibial fatigue fracture has been shown to increase by a factor of 1.4 for every $1 \mathrm{~g}$ increase in TA. ${ }^{4,10}$

In most geographic locales, runners encounter terrain of varying grade and surface type, which may alter TA and the risk for overuse injuries. ${ }^{12,13}$ In regard to grade, higher impact peaks have been observed during decline running and lower impact forces have been observed during incline running when compared

Waite is with the Exercise Science \& Athletic Training Department, Adrian College, Adrian, MI, USA. Goetschius is with the Department of Health Professions, James Madison University, Harrisonburg, VA, USA. Lauver is with the Department of Kinesiology, Coastal Carolina University, Conway, SC, USA. Lauver (jlauver@ coastal.edu) is corresponding author. with level running on a treadmill. ${ }^{12}$ In addition, softer surfaces such as rubberized surfaces have been associated with lower impact forces compared with hard surfaces such as asphalt. ${ }^{14,15}$ However, to our knowledge, no previous investigation has examined the effect of surface type on TA during incline and/or decline running in a real-world setting. Therefore, the aim of this study was to compare peak TA in trained distance runners on 3 surface types (grass, asphalt, and concrete) and 3 grades (incline, decline, and level). First, the authors hypothesized that runners would experience lower peak TA on a grass surface compared with the harder surfaces of asphalt and concrete during all grades. Second, the authors hypothesized that decline running would result in higher peak TA compared with incline and level grades.

\section{Methods}

This study involved a crossover design with counterbalanced conditions. A total of 13 trained distance runners $(8$ males and 5 females, age $=20.07 \quad[0.95] \quad \mathrm{y}$, height $=1.73[0.09] \mathrm{m}$, weight $=62.18$ $[7.88] \mathrm{kg}$ ) volunteered to participate in this study (Table 1). Results from a power analysis utilizing a moderate effect size (0.5), alpha of .05 , and power of 0.80 indicated that a sample size of 9 would meet sufficient statistical power. ${ }^{16}$ Subjects were fully informed of the purpose, risk, and discomforts associated with the study before providing written consent. The study complied with the standards set by the Declaration of Helsinki and was approved by the Adrian College Institutional Review Board. Based on previous research, the ${ }^{6,10}$ inclusion criteria included: running at least 25 to 30 miles per week for females and 35 to 40 miles a week for males. Any individual who self-reported a history of metabolic, pulmonary, cardiovascular disease, and/or an orthopedic-related injury in the past 6 months was excluded.

\section{Procedures}

During visit 1, subjects performed a maximal effort 1-mile time trial. This time was used to determine a running velocity that 
corresponded to $80 \%$ of the average velocity during the time trial. This $80 \%$ aligns with a running velocity that would typically be utilized during a tempo training session. Before the time trial, subjects completed a 5-minute self-selected warm-up. After the time trial, subjects completed practice trials to become familiarized with the running velocity for session 2 .

During visit 2, a skin-mounted accelerometer (Trigno; Delsys Inc, Natick, MA) was attached to the distal anterior-medial aspect of each subject's tibia, $8 \mathrm{~cm}$ above the medial malleolus of the dominant leg. The axis of the accelerometer was aligned with the long axis of the subject's tibia ${ }^{17}$ to collect data in the vertical axis; therefore, all accelerometer data presented are vertical axial acceleration. To limit unwanted movement of the accelerometer, the site of attachment was shaved, double-sided adhesive tape was applied, and elastic nonadhesive tape was lightly wrapped around the accelerometer. Subjects completed a 5-minute self-selected warm-up. In a randomized order, subjects completed running trials on 3 different surface types (grass, asphalt, and concrete) and 3 different grades (incline, decline, and level); the incline and decline grades were at $4^{\circ}$. All running trials were conducted outdoors on surfaces that are typically experienced during real-world running (eg, outside the laboratory). The location utilized for the incline and decline grades was selected due to its consistent grade and inclusion of all surface types under investigation. In addition, the location was selected due to its proximity to the level grade condition location to minimize any variations in surface types investigated. The asphalt condition consisted of typical hot asphalt used in road construction, and the concrete condition was concrete typically used for walkways. Subjects ran 2 trials for each surface and grade condition, for a total of 18 trials. For each trial, the subjects ran a total of $20 \mathrm{~m}$ at $80 \%$ of the average velocity from the time trial. Subjects were provided with a $10-\mathrm{m}$ run-up and were asked to achieve the $80 \%$ velocity before reaching the running zone, which was identified with cones. The 10-m run-up and 20-m area of investigation both comprised consistent grades and surface conditions under investigation. Trials were discarded and repeated if there was a $\pm 5 \%$ difference in velocity until 2 successful trials were performed for each combination. A video camera (C615 Logitech HD; Logitech Inc, Newark, CA) was used to simultaneously record with the TA and was used to assess velocity during all trials. Velocity was calculated utilizing the time taken to cover the 20-m area of investigation from the video recording. Between all running trials, subjects were given 2 minutes of rest to minimize the effect of fatigue.

\section{Data Processing}

All TA data were collected on LabChart 8 (ADInstruments, Colorado Springs, CO) and processed using a Butterworth low-pass zero-lag filter with a cutoff frequency of $60 \mathrm{~Hz} .{ }^{4}$ The synchronized video footage was used to identify the first 4 stance phases with the dominant foot in the middle $10 \mathrm{~m}$ of each trial. The peak TA during

\section{Table 1 Subjects Training Descriptives, Mean (SD)}

\begin{tabular}{lc}
\hline Miles training per week & $52.69(10.73)$ \\
Days training per week & $6.38(0.77)$ \\
Years of training & $5.73(2.24)$ \\
Time trial time, s & $328.2(35.9)$ \\
Trial velocity, m/s & $3.94(0.42)$ \\
\hline
\end{tabular}

each stance phase was recorded, resulting in 8 peak TA values per grade and surface combination, which were then averaged to determine the average peak TA.

\section{Statistical Analysis}

A 2-way $(3 \times 3)$ analysis of variance with repeated-measures and post hoc Tukey multiple comparison tests were used to determine the effects of surface (grass, asphalt, and concrete) and grade (incline, decline, and level) on peak TA. Statistical significance was set a priori at $P \leq .05$. Unless otherwise stated, all values are expressed as mean (SD). Cohen $d$ effect sizes $(d)$ and 95\% confidence intervals (95\% CIs) were also calculated. Effect size interpretations were $<0.2=$ minimal, 0.2 to $0.5=$ small, 0.5 to $0.8=$ moderate, and $>0.8=$ large, and $95 \%$ CIs not including 0 were interpreted as clinically important. Data were analyzed with SigmaPlot software (SigmaPlot 14; Systat Software, Inc, San Jose, CA).

\section{Results}

There was no significant main effect for surface $(P=.085)$; however, there was a significant interaction $(P=.05)$ (Figure 1$)$; on level grade, the grass resulted in higher peak TA compared with concrete $(P=.017, d=0.52)$. During the incline grade, grass resulted in higher peak TA compared with asphalt $(P=.030$, $d=0.45)$. There was a significant main effect for grade $(P=.021)$ with decline (8.04 [1.36] g; 95\% CI, 7.59-8.49), resulting in higher peak TA compared with incline $(7.31$ [1.58] g; 95\% CI, 6.79-7.83; $P=.020 ; d=0.48)$. There was no difference in peak TA during level grade (7.83 [1.42] g; 95\% CI, 7.36-8.29) compared with incline $(P=.120)$. Furthermore, post hoc analysis of the significant interaction showed that decline running on asphalt surface resulted in higher peak TA compared with incline on asphalt $(P=.001$, $d=0.67$ ). Level grade running on asphalt surface resulted in higher peak TA compared with incline on asphalt $(P=.030, d=0.51)$. Means and SDs are presented in Table 2.

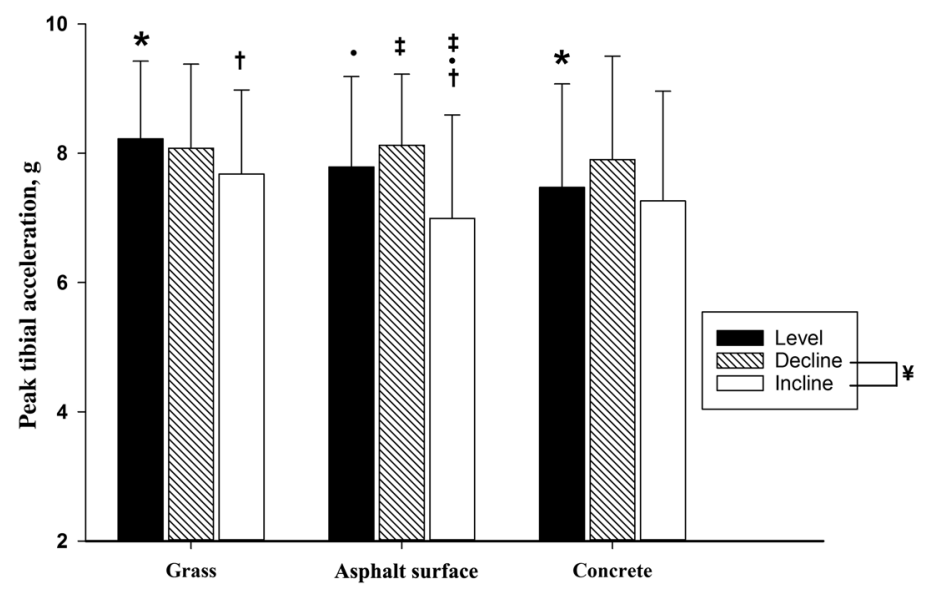

Figure 1 - Peak tibial acceleration means (SD). ${ }^{\sharp}$ Significant difference between decline and incline on all surfaces. *Significant difference between grass and concrete on the level grade. $†$ Significant difference between grass and asphalt on the incline grade. $\ddagger$ Significant difference between decline and incline on the asphalt surface. • Significant difference between level and incline on asphalt. 
Table 2 Peak Tibial Acceleration Means (SD) and 95\% Confidence Intervals (Cl)

\begin{tabular}{|c|c|c|c|c|c|c|}
\hline \multirow[b]{2}{*}{ Grade } & \multicolumn{2}{|c|}{ Grass } & \multicolumn{2}{|c|}{ Asphalt } & \multicolumn{2}{|c|}{ Concrete } \\
\hline & Mean (SD) & $95 \% \mathrm{Cl}$ & Mean (SD) & $95 \% \mathrm{Cl}$ & Mean (SD) & $95 \% \mathrm{Cl}$ \\
\hline Incline & $7.68(1.35)^{* *}$ & $6.86-8.50$ & 6.99 (1.69) & $5.97-8.02$ & $7.26(1.78)$ & $6.19-8.34$ \\
\hline Decline & $8.08(1.36)$ & $7.25-8.90$ & $8.12(1.17)^{* * *}$ & $7.42-8.83$ & $7.90(1.66)$ & $6.90-8.91$ \\
\hline Level & $8.22(1.22)^{*}$ & $7.49-8.96$ & $7.79(1.44)^{\#}$ & $6.92-8.66$ & $7.47(1.65)$ & $6.47-8.47$ \\
\hline
\end{tabular}

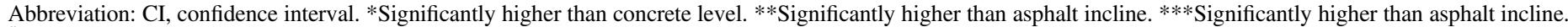

\#Significantly higher than asphalt incline.

\section{Discussion}

The aim of the current investigation was to compare peak TA in trained distance runners on 3 surface types (grass, asphalt, and concrete) and 3 grades (incline, decline, and level). Contrary to our first hypothesis, trained runners experienced higher peak TA on grass compared with concrete on the level surface and on grass compared with asphalt on the incline surface. In contrast to our findings, previous investigations have found that running on softer surfaces such as a rubberized surface results in lower GRF and TA compared with harder surfaces. ${ }^{14,15,18,19}$ However, previous investigations have utilized synthetic tracks and turf, ${ }^{1,15}$ which are different than the grass condition in the current investigation. The natural grass surface may result in more imperfections in the surface compared with other artificial surfaces. These imperfections could have resulted in kinematic alterations in the current investigation, which may have affected the peak TA. However, other investigations have demonstrated that peak TA was not different between surfaces of varying hardness. ${ }^{1} \mathrm{~A}$ factor possibly contributing to the differences observed between studies is the running velocity utilized. Running at higher velocities has been demonstrated to result in higher TA on hard surfaces compared with softer surfaces ${ }^{18}$; however, at slower velocities, TA may not be different between surfaces. ${ }^{1,18}$ In addition, although subjects in the current investigation ran at a velocity dependent on their timetrial performance, previous investigations have utilized a fixed velocity for all participants. ${ }^{1,15,18}$ The utilization of a fixed velocity may result in participants running at different percentages of their maximal performance, which may potentially affect TA, therefore making comparisons between investigations more difficult.

Although the surface condition is one factor that can affect peak TA, other factors, such as the knee and ankle angle at ground contact and muscle preactivation (activation before ground contact), need to be considered. ${ }^{20}$ During running, muscles, tendons, and ligaments are utilized to store and return elastic energy as a single linear "leg spring," ${ }^{21}$ which is affected by several factors, including the knee and ankle angle, foot striking pattern, and muscle activity. Muscle activity is associated with several tasks prior to ground contact, including positioning the leg, developing joint stiffness, and controlling soft tissue compartment vibrations. ${ }^{22,23}$ Joint stiffness is adjusted during running on different surfaces so that a runner can maintain a constant stride frequency and contact time. ${ }^{24}$ To maintain these factors on softer surfaces, runners typically run with a straighter leg, resulting in increased joint stiffness, ${ }^{24}$ possibly resulting in an increase in TA. The subjects in this study were trained distance runners and may have perceived that the grass surface would be the softest of the 3 surfaces based on experience and with that perception may have landed with a stiffer leg, thus resulting in higher TA.

In agreement with our second hypothesis, incline resulted in lower TA compared with decline. Previous evidence suggests that incline running is also associated with a higher metabolic cost of running compared with decline, 25 and decline running is associated with greater levels of muscle damage. ${ }^{26}$ Contrary to our second hypothesis, the decline did not result in a higher TA compared with the level grade. Although previous investigations have demonstrated higher impact peaks during decline compared with level running,,$^{12}$ the lack of differences between grades observed in the current investigation may have been due to the degree of the grade utilized. As the degree of a decline increases, the impact peak can increase by $54 \%$ at a $-9^{\circ}$ angle compared with level running. ${ }^{12}$ However, as the angle decreases so does the difference in TA between decline and level running. ${ }^{12,27}$ The lack of difference in the peak TA between decline and level grades observed in the current investigation may have been due to the $4^{\circ}$ grade not being large enough to produce a difference in peak TA. The current investigation was limited by the geography of the study location; therefore, future investigations should utilize a grade greater than the $4^{\circ}$.

The current investigation is not without limitations. First, the grass surface was a strip of grass located between the asphalt and concrete surfaces. Although this was selected to mimic typical running conditions and to ensure a similar grade for all surface conditions, it may have resulted in the grass surface being harder than other grass surfaces (eg, in an open field) due to increased foot traffic and/or the construction of either the asphalt or the concrete surfaces. A further potential limitation to the current investigation was the foot-striking pattern of the subjects. Subjects were not selected based on any specific foot-striking pattern; therefore, varying running styles may have affected the peak TA. However, our crossover design would have helped control for variability in running styles. In addition, the impact that grade and/or surface had on the foot-striking pattern was not evaluated in the current investigation but warrants further consideration.

The current study demonstrated that under certain grades a grass surface may result in higher TA then asphalt or concrete. In addition, incline running on a moderate grade results in lower peak TA compared with decline. Therefore, consideration should be given to the surface and grade experienced during distance running, as the cumulative effect of the repeated impact forces can impact the development of overuse injuries. In conclusion, the results of this study may be beneficial to athletes and coaches when designing training programs to help reduce the peak TA experienced by runners.

\section{Acknowledgments}

This study was conducted without any financial support, and the authors have no conflicts of interest to disclose.

\section{References}

1. Fu WJ, Fang Y, Liu DMS, Wang L, Ren SC, Liu Y. Surface effects on in-shoe plantar pressure and tibial impact during running. J Sport Health Sci. 2015;4(4):384-390. doi:10.1016/j.jshs.2015.09.001 
2. Hreljac A, Marshall RN, Hume PA. Evaluation of lower extremity overuse injury potential in runners. Med Sci Sports Exerc. 2000; 32(9):1635-1641. PubMed ID: 10994917 doi:10.1097/00005768200009000-00018

3. Nigg BM. The role of impact forces and foot pronation: a new paradigm. Clin J Sport Med. 2001;11(1):2-9. PubMed ID: 11176139 doi:10.1097/00042752-200101000-00002

4. Sheerin KR, Reid D, Besier TF. The measurement of tibial acceleration in runners-a review of the factors that can affect tibial acceleration during running and evidence-based guidelines for its use. Gait Posture. 2019;67:12-24. PubMed ID: 30248663 doi:10.1016/j. gaitpost.2018.09.017

5. Pohl MB, Mullineaux DR, Milner CE, Hamill J, Davis IS. Biomechanical predictors of retrospective tibial stress fractures in runners. J Biomech. 2008;41(6):1160-1165. PubMed ID: 18377913 doi:10.1016/j.jbiomech.2008.02.001

6. Clansey AC, Lake MJ, Wallace ES, Feehally T, Hanlon M. Can trained runners effectively attenuate impact acceleration during repeated high-intensity running bouts? J Appl Biomech. 2016;32(3): 261-268. PubMed ID: 26695109 doi:10.1123/jab.2015-0125

7. Whittle MW. Generation and attenuation of transient impulsive forces beneath the foot: a review. Gait Posture. 1999;10(3):264-275. PubMed ID: 10567759 doi:10.1016/S0966-6362(99)00041-7

8. Mizrahi J, Verbitsky O, Isakov E. Fatigue-related loading imbalance on the shank in running: a possible factor in stress fractures. Ann Biomed Eng. 2000;28(4):463-469. PubMed ID: 10870903 doi:10. $1114 / 1.284$

9. Hennig EM, Lafortune MA. Relationships between ground reaction force and tibial bone acceleration parameters. Int J Sport Biomech. 1991;7(3):303-309. doi:10.1123/ijsb.7.3.303

10. Milner CE, Ferber R, Pollard CD, Hamill J, Davis IS. Biomechanical factors associated with tibial stress fracture in female runners. Med Sci Sports Exerc. 2006;38(2):323-328. PubMed ID: 16531902 doi:10. 1249/01.mss.0000183477.75808.92

11. Lafortune MA, Henning E, Valiant GA. Tibial shock measured with bone and skin mounted transducers. J Biomech. 1995;28(8):989-993. PubMed ID: 7673266 doi:10.1016/0021-9290(94)00150-3

12. Gottschall JS, Kram R. Ground reaction forces during downhill and uphill running. J Biomech. 2005;38(3):445-452. PubMed ID: 15652542 doi:10.1016/j.jbiomech.2004.04.023

13. Tessutti V, Ribeiro AP, Trombini-Souza F, Sacco IC. Attenuation of foot pressure during running on four different surfaces: asphalt, concrete, rubber, and natural grass. J Sports Sci. 2012;30(14): 1545-1550. PubMed ID: 22897427 doi:10.1080/02640414.2012. 713975

14. Dixon SJ, Collop AC, Batt ME. Surface effects on ground reaction forces and lower extremity kinematics in running. Med Sci Sports Exerc. 2000;32(11):1919-1926. doi:10.1097/00005768-20001100000016
15. Ueberschär O, Fleckenstein D, Warschun F, Kränzler S, Walter N, Hoppe MW. Measuring biomechanical loads and asymmetries in junior elite long-distance runners through triaxial inertial sensors. Sports Orthop Traumatol. 2019;35(3):296-308. doi:10.1016/j.orthtr. 2019.06.001

16. Faul F, Erdfelder E, Buchner A, Lang AG. Statistical power analyses using $\mathrm{G}^{*}$ Power 3.1: tests for correlation and regression analyses. Behav Res Methods. 2009;41(4):1149-1160. PubMed ID: 19897823 doi:10.3758/BRM.41.4.1149

17. Crowell HP, Davis IS. Gait retraining to reduce lower extremity loading in runners. Clin Biomech. 2011;26(1):78-83. PubMed ID: 20888675 doi:10.1016/j.clinbiomech.2010.09.003

18. Greenhalgh A, Sinclair J, Leat A, Chockalingam N. Influence of footwear choice, velocity and surfaces on tibial accelerations experienced by field hockey participants during running. Footw Sci. 2012;4(3):213-219. doi:10.1080/19424280.2012.696725

19. Boey H, Aeles J, Schutte K, Vanwanseele B. The effect of three surface conditions, speed and running experience on vertical acceleration of the tibia during running. Sports Biomech. 2017;16(2):166176. PubMed ID: 27595311 doi:10.1080/14763141.2016.1212918

20. Potthast W, Bruggemann GP, Lundberg A, Arndt A. The influences of impact interface, muscle activity, and knee angle on impact forces and tibial and femoral accelerations occurring after external impacts. $J$ Appl Biomech. 2010;26(1):1-9. PubMed ID: 20147752 doi:10.1123/ jab.26.1.1

21. Ferris DP, Louie M, Farley CT. Running in the real world: adjusting leg stiffness for different surfaces. Proc Biol Sci. 1998;265(1400): 989-994. PubMed ID: 9675909 doi:10.1098/rspb.1998.0388

22. Nigg BM, Wakeling JM. Impact forces and muscle tuning: a new paradigm. Exerc Sport Sci Rev. 2001;29(1):37-41. doi:10.1097/ 00003677-200101000-00008

23. Boyer KA, Nigg BM. Changes in muscle activity in response to different impact forces affect soft tissue compartment mechanical properties. J Biomech Eng. 2007;129(4):594-602. PubMed ID: 17655481 doi: $10.1115 / 1.2746384$

24. Ferris DP, Liang K, Farley CT. Runners adjust leg stiffness for their first step on a new running surface. J Biomech. 1999;32(8):787-794. PubMed ID: 10433420 doi:10.1016/S0021-9290(99)00078-0

25. Vernillo G, Savoldelli A, Zignoli A, et al. Energy cost and kinematics of level, uphill and downhill running: fatigue-induced changes after a mountain ultramarathon. J Sports Sci. 2015;33(19):1998-2005. PubMed ID: 25751128 doi:10.1080/02640414.2015.1022870

26. Eston RG, Mickleborough J, Baltzopoulos V. Eccentric activation and muscle damage: biomechanical and physiological considerations during downhill running. Br J Sports Med. 1995;29(2):89-94. PubMed ID: 7551767 doi:10.1136/bjsm.29.2.89

27. Mizrahi J, Verbitsky O, Isakov E. Shock accelerations and attenuation in downhill and level running. Clin Biomech. 2000;15(1):15-20. PubMed ID: 10590340 doi:10.1016/S0268-0033(99)00033-9 